\title{
COVID-19-Pneumonie mit Lungenultraschall schnell erkennen und Klinikpersonal schützen
}

Berlin - Schwere Verläufe der COVID-19Erkrankung gehen mit einer Lungenentzündung einher. Symptome wie Fieber, starker Husten und häufig auch Luftnot kennzeichnen die Infektion. Doch wie lässt sich schnell und sicher erkennen, ob eine Pneumonie oder eine andere Lungenerkrankung vorliegt? Gibt es möglicherweise Hinweise darauf, dass es sich um eine Infektion mit dem Coronavirus handelt? Wie entwickelt sich die Erkrankung im weiteren Verlauf einer stationären Behandlung? Nach Ansicht der Experten der Deutschen Gesellschaft für Ultraschall in der Medizin e. V. (DEGUM) ist für diese Fragestellungen ein Lungenultraschall ideal geeignet. Die Thoraxsonografie ist schnell und direkt am Krankenbett einsetzbar sowie beliebig oft wiederholbar. Da nur 1 Arzt die Diagnostik durchführt, werden zudem Kontakte zwischen Patienten und Klinikpersonal stark reduziert.

„Ein Point-of Care-Ultraschall - PoCUS - der Lunge ist ein sehr wertvolles und effektives Instrument, um den Zustand der Lunge eines Patienten schnell und sicher einzuschätzen“, sagt Professor Dr. med. Josef Menzel, Direktor der Medizinischen Klinik II am Klinikum Ingolstadt und Neupräsident der DEGUM. Neben den überall verfügbaren Ultraschallsystemen eignen sich portable Geräte, die leicht zu desinfizieren sind, hierfür besonders. So können die Anwender Ultraschall direkt am Krankenbett einsetzen, ohne den Patienten verlegen zu müssen. Auch die hygienischen Voraussetzungen bleiben gewahrt, da die kleinen tragbaren Geräte für die Untersuchung in Tüten verpackt werden können. „Der Vorteil an diesem Verfahren ist, dass der gleiche Arzt, der den Patienten aufgenommen hat, auch die Sonografie am Bett durchführen kann das führt zu einer erheblichen Reduzierung weiterer Kontaktpersonen“, hebt Menzel hervor.

Der Standard sieht vor, dass Patienten mit Verdacht auf COVID-19-Pneumonie im Röntgen oder im CT untersucht werden. Zusammen mit dem klinischen Bild des Patienten könne der Ultraschall jedoch Weichen stellen, ob eine weitere bildgebende Untersuchung unverzüglich erfolgen muss oder erst im Verlauf der Behandlung eingesetzt werden kann, erklärt Menzel. „Bedenkt man, wie lange ein Patient in einer Notaufnahme auf eine Röntgen- oder CT-Untersuchung warten muss, so liegt auch der Zeitvorteil des Ultraschalls klar auf der Hand." Auch während der stationären Behandlung werden wiederholt Röntgen-Thorax-Aufnahmen durchgeführt. Hier könne die Lungensonografie morphologische Veränderungen in der Lunge einfach, schnell und sicher feststellen oder auch ausschließen - und so im Einzelfall die Frequenz der Röntgen-Untersuchungen reduzieren.

Zum Einsatz des PoCUS-Lungenultraschalls bei COVID-19-Patienten gibt es bereits erste Publikationen aus China und Italien. „Wir erhalten mithilfe der Thoraxsonografie zwar keinen abschließenden Beweis für eine COVID-19-Pneumonie, es gibt aber klar erkennbare Muster, die für eine Viruspneumonie sprechen. Diese typischen Veränderungen des Lungenbildes erlauben auch eine Verlaufsbeurteilung“, erläutert Dr. Alexander Heinzmann, Leitender Oberarzt am Klinikum am Steinenberg in Reutlingen und Leiter des Arbeitskreises Thoraxsonografie der DEGUM. „Die DEGUM plant gerade eine Sammlung typischer Bildbefunde, die die Kolleginnen und Kollegen demnächst auf der Homepage der DEGUM einsehen und als Orientierungshilfe nutzen können“, ergänzt Menzel.

„Bei Patienten mit respiratorischer Insuffizienz sowie dem Verdacht auf eine Lungenentzündung sollte immer sofort ein Lungenultraschall durchgeführt werden“, so das Fazit von Heinzmann. Ultraschall ist frei von Strahlung, schnell, günstig und sicher und gehört in diesen Zeiten in jede Notaufnahme und auf jede Intensivstation, so die Experten abschließend.

\section{Weiterführende Informationen:}

Buonsenso D et al. Point-of-Care Lung Ultrasound findings in novel coronavirus disease-19 pnemoniae: a case report and potential applications during COVID-19 outbreak. Eur Rev Med Pharmacol Sci. 2020 Mar; 24:2776-2780

Qian-Yi Peng et al. Findings of lung ultrasonography of novel corona virus pneumonia during the 2019-2020 epidemic. Intensive Care Med. 2020 May; 46:849-850. doi: 10.1007/s00134-020-05996-6. Epub 2020 Mar 12. 